\title{
Datengetriebene Geschäftsmodelle: Konzeptuelles Rahmenwerk, Praxisbeispiele und Forschungsausblick
}

\author{
Susanne Strahringer $(\mathbb{D}) \cdot$ Martin Wiener $(\mathbb{D})$
}

Eingegangen: 28. Februar 2021 / Angenommen: 2. April 2021 / Online publiziert: 25. April 2021

(C) Der/die Autor(en) 2021

Zusammenfassung Datengetriebene Geschäftsmodelle (DGGM) vereinen zwei zentrale Trends des digitalen Zeitalters: die steigende Bedeutung von (,Big“-)Daten und den zunehmenden Fokus auf das Geschäftsmodell als zentrale Gestaltungsebene. Nichtsdestotrotz sind die konzeptuellen Grundlagen und spezifischen Eigenschaften entsprechender Geschäftsmodelle bislang nur unzureichend erforscht und systematisiert. Basierend auf relevanten Vorarbeiten stellen wir in diesem Grundlagenbeitrag drei wesentliche Typen von DGGM vor (Datennutzer, Datenlieferanten und Datenbefähiger) und geben einen Überblick über zentrale „,datengetriebene“ Konzepte, die wir in ein konzeptuelles Rahmenwerk zur weiterführenden Charakterisierung von DGGM integrieren. Die Anwendbarkeit und Nützlichkeit der drei DGGM-Typen und des entwickelten Rahmenwerks illustrieren wir anhand von drei Praxisbeispielen (KAESER, Shell Telematics und Celonis). Der Beitrag schließt mit einer Diskussion von vielversprechenden, thematischen Ansatzpunkten für zukünftige Forschungsarbeiten zu DGGM.

Schlüsselwörter Datengetriebene Geschäftsmodelle $\cdot$ Typologie $\cdot$ Konzeptuelles Rahmenwerk · Praxisbeispiele · Zukünftige Forschungsrichtungen

\footnotetext{
S. Strahringer $(\square)$

Lehrstuhl für Wirtschaftsinformatik, insbes. Informationssysteme in Industrie und Handel, TU Dresden, Helmholtzstr. 10, 01069 Dresden, Deutschland

E-Mail: Susanne.Strahringer@tu-dresden.de

M. Wiener

Lehrstuhl für Wirtschaftsinformatik, insbes. Business Engineering, TU Dresden,

Helmholtzstr. 10, 01069 Dresden, Deutschland

E-Mail: Martin.Wiener@tu-dresden.de
} 


\title{
Data-driven Business Models: Conceptual Framework, Practical Examples, and Research Outlook
}

\begin{abstract}
Data-driven business models (DDBMs) combine two central trends of the digital era: the growing importance of ("big") data and the increasing focus on the business model as the focal design layer. Nevertheless, thus far, the conceptual foundations and specific characteristics of corresponding business models have been only insufficiently researched and systematized. Based on relevant prior works, in this foundations paper, we introduce three main types of DDBMs (data users, data suppliers, and data facilitators) and provide an overview of key "data-driven" concepts, which we integrate into a conceptual framework for characterizing DDBMs. To illustrate the applicability and usefulness of the three DDBM types and the developed framework, we present three real-world examples (KAESER, Shell Telematics, and Celonis). The paper concludes with a discussion of promising directions for future research on DDBMs.
\end{abstract}

Keywords Data-driven business models - Typology · Conceptual framework · Practical examples $\cdot$ Future research directions

\section{Einführung}

Datengetriebene Geschäftsmodelle (DGGM) vereinen zwei zentrale Trends des digitalen Zeitalters: die steigende Bedeutung von Daten für Organisationen (Chen et al. 2012) und den zunehmenden Fokus auf das Konzept des Geschäftsmodells im Rahmen der Analyse und Gestaltung von Organisationen (Veit et al. 2014). Zum einen haben digitale Technologien wie Smartphones und soziale Medien sowie der verstärkte Einsatz von Sensorik in „smarten“ Produkten und Maschinen zu einem exponentiellen Wachstum des weltweit verfügbaren Datenvolumens geführt (Hartmann et al. 2016). In diesem Zusammenhang werden Daten oftmals als das „,neue Öl“ bezeichnet (Rotella 2012). Auch wenn die Analogie zwischen Öl und Daten teils ,hinkt“ (z.B. im Hinblick auf die nahezu unbegrenzte Verfügbarkeit und Wiederverwendbarkeit von Datenressourcen im Gegensatz zur knappen und endlichen Ressource Öl), so erscheint sie im Kern doch recht treffend: sowohl Daten als auch Öl verfügen über ein enormes Wertschöpfungspotenzial und beide müssen zunächst be- bzw. verarbeitet werden, bevor sie ihr Potenzial entfalten können (Hartmann et al. 2016).

Zum anderen gewinnt das Geschäftsmodellkonzept seit Ende der 1990er Jahre sowohl in der Forschung als auch in der der Unternehmenspraxis zunehmend an Interesse und Bedeutung (Al-Debei und Avison 2010; Bock und Wiener 2017). In der Praxis wird das Geschäftsmodell zum Beispiel als zentrales „Werkzeug“ zur Beschreibung, Erneuerung und/oder Bewertung der grundlegenden Geschäftslogik von Start-ups und etablierten Organisationen gesehen (Veit et al. 2014). In der Forschung wird das Geschäftsmodellkonzept insbesondere als ein „Alignment-Werkzeug“"verstanden, das die aus dem schnellen technologischen Wandel und dem damit einhergehenden Komplexitätsanstieg resultierende „klaffende“ Lücke zwi- 
schen Geschäftsstrategie und deren Umsetzung auf Prozessebene schließt (Al-Debei und Avison 2010). Demzufolge argumentieren Forscher, dass das Geschäftsmodell die zentrale Untersuchungsebene einer neuen Forschungsära darstellen könnte (Veit et al. 2014) und dass der Wettbewerb zwischen Organisationen zukünftig vor allem auf der Geschäftsmodellebene entschieden wird (Gassmann et al. 2014).

Vor diesem Hintergrund ist es kaum überraschend, dass derzeit eine Vielzahl von Organisationen - über Branchen- und Ländergrenzen hinweg - versuchen, das erhebliche Potenzial von (,Big“-)Daten zur Generierung von neuen bzw. zur Aufrechterhaltung von bestehenden Wettbewerbsvorteilen zu nutzen, indem sie ihre traditionellen Geschäftsmodelle mit datengetriebenen Elementen anreichern oder vollständig neue DGGM entwickeln (Wiener et al. 2020). Der potenzielle Mehrwert entsprechender Bestrebungen ist Studien zufolge beachtlich. Zum Beispiel zeigt eine Studie des Massachusetts Institute of Technology (MIT), dass datengetriebene Organisationen eine höhere Produktivität und Profitabilität aufweisen als andere Organisationen (Brynjolfsson et al. 2011). Ein weiteres Beispiel für das mit DGGM verbundene Wertschöpfungspotenzial zeigt sich auch bei genauerer Betrachtung der aktuellen Liste der ,world's most valuable brands“ (Forbes 2020). Während die Forbes-Liste vor 20 Jahren - also zu Beginn des digitalen Zeitalters - noch von Ölfirmen (Exxon Mobil) und Automobilkonzernen (General Motors und Ford Motor) dominiert wurde, so wird diese Liste mittlerweile von Technologiefirmen (Apple, Google, Microsoft, Amazon, Facebook) angeführt, in deren Geschäftsmodellen Daten eine zentrale Rolle spielen. Zum Beispiel erwirtschaftet Google 80-90\% seines Jahresumsatzes mit datengetriebener Werbung, was im Jahr 2020 circa 155 Mrd. US-Dollar entsprach. Im Vergleich hierzu lag der Gesamtumsatz von großen DAXKonzernen bei rund 120 Mrd. US-Dollar (BMW) bzw. bei 70 Mrd. US-Dollar (Siemens). Dieses Beispiel soll aber nicht suggerieren, dass sich die zentrale Rolle von Daten auf die Geschäftsmodelle von Firmen der sog. „New Economy“ beschränkt; vielmehr spielen Daten auch zunehmend eine bedeutsame Rolle in den Geschäftsmodellen zahlreicher traditioneller Unternehmen der „Old Economy“. Exemplarisch können hier Firmen wie z. B. adidas, KAESER, Shell und Siemens genannt werden.

Trotz der zunehmenden Bedeutung und Popularität von DGGM in Praxis und Forschung sind die konzeptuellen Grundlagen entsprechender Geschäftsmodelle bislang nur unzureichend erforscht und systematisiert (Hartmann et al. 2016; Wiener et al. 2020). Insbesondere fehlt es an konzeptuellen Rahmenwerken, die die Besonderheiten von DGGM (im Vergleich zu herkömmlichen Geschäftsmodellen) angemessen berücksichtigen. Genau an dieser Stelle setzt der vorliegende Grundlagenbeitrag an, indem er zunächst Basiskonzepte in Bezug auf Geschäftsmodelle im Allgemeinen und Dateneigenschaften einführt und auf dieser Basis dann zentrale ,datengetriebene“ Konzepte aus relevanten Vorarbeiten in ein konzeptuelles Rahmenwerk für DGGM integriert. Nach einer Illustration des vorgestellten Rahmenwerks anhand von drei Praxisbeispielen schließt der Beitrag mit einer Diskussion von vielversprechenden Möglichkeiten für zukünftige Forschungsarbeiten zu DGGM. 


\section{Konzeptuelle Grundlagen}

\subsection{Das Geschäftsmodell als zentrale Gestaltungsebene}

Das Geschäftsmodellkonzept im Allgemeinen dient der Beschreibung der wesentlichen Geschäftslogik einer Organisation (Osterwalder et al. 2005). Der Fokus liegt hierbei allen voran auf der Frage, wie eine Organisation Wert schafft und auch realisiert; d.h. vom geschaffenen Wert selbst ,profitiert“ (Osterwalder und Pigneur 2010). In diesem Zusammenhang wird das Geschäftsmodell oftmals als neue und zentrale Gestaltungsebene verstanden, die sich kritisch auf den Geschäftserfolg einer Organisation auswirkt (Veit et al. 2014) und somit bestehende Ansätze zur Analyse und Gestaltung von Organisationen sinnvoll erweitert (Al-Debei und Avison 2010). Exemplarisch können an dieser Stelle etablierte Architektur- und Modellierungsansätze des Business Engineering (wie z. B. ARIS und der St. Galler Ansatz) genannt werden, die typischerweise auf drei wesentliche Gestaltungsebenen fokussieren: Geschäftsstrategie, Geschäftsprozesse sowie Informations- und Kommunikationssysteme (Österle und Blessing 2005). Aufgrund der aus der aktuellen digitalen Transformation von Organisationen resultierenden und sich nach wie vor ausweitenden „Kluft“ zwischen Geschäftsstrategie und -prozessen wird dieser eingeschränkte Fokus auf nur drei Gestaltungsebenen zunehmend als nicht mehr ausreichend und zeitgemäß erachtet (Al-Debei und Avison 2010).

Eine in Forschung und Praxis gleichermaßen akzeptierte und weit verbreitete Konzeptualisierung des Geschäftsmodellkonzepts findet sich in Osterwalder und Pigneur (2010). Diese unterscheidet zwischen neun wesentlichen Bausteinen bzw. Elementen zur Beschreibung des Geschäftsmodells einer Organisation: Wertangebote (d.h. Produkte und/oder Services), Kundensegmente, -beziehungen und -kanäle, Kernaktivitäten und -ressourcen, Schlüsselpartner sowie Kostenstruktur und Umsatzquellen. Diese Elemente lassen sich nach Al-Debei und Avison (2010) vier zentralen Geschäftsmodelldimensionen (,4 Ws“) zuordnen (siehe Abb. 1): (1) das Wertversprechen beschreibt die Produkte und/oder Services einer Organisation und deren Zielgruppen (Kundensegmente) sowie die Interaktion mit den adressierten Kundengruppen (Beziehungen und Kanäle); (2) die Wertarchitektur bezieht sich auf die organisationalen Kernaktivitäten und -ressourcen, die zur Erstellung der angebotenen Produkte und/oder Services erforderlich sind; (3) das Wertnetzwerk bezeichnet

Abb. 1 ,4 W"-Geschäftsmodelldimensionen. (Nach AlDebei und Avison 2010)

\begin{tabular}{cc|}
$\begin{array}{c}\text { Wertversprechen } \\
\text { ("value proposition") }\end{array}$ & $\begin{array}{c}\text { Wertarchitektur } \\
\text { ("value architecture") }\end{array}$ \\
$\begin{array}{c}\text { Produkte und/oder Services } \\
\text { Kundensegmente }\end{array}$ & $\begin{array}{c}\text { Kernaktivitäten } \\
\text { Kernressourcen }\end{array}$ \\
Kundenbeziehungen und Kanäle & Wertrealisierung \\
("value finance") \\
Wertnetzwerk \\
("value network") \\
Beziehungen zu \\
und Interaktionen mit \\
(externen) Schlüsselpartnern
\end{tabular}


insbesondere die Beziehungen zu und Interaktionen mit (externen) Schlüsselpartnern, die zur Erstellung des Wertangebots notwendig sind; (4) die Wertrealisierung umfasst die Kostenstruktur und die wesentlichen Umsatzquellen einer Organisation.

Die „4 W“-Dimensionen und die zugehörigen Geschäftsmodellelemente sind grundsätzlich auch auf DGGM anwendbar; d.h. DGGM-spezifische Konzepte lassen sich auf diese Dimensionen und (ausgewählte) Elemente abbilden. Auch aus Gründen der Kontinuität und Vergleichbarkeit wird sich daher das in Abschnitt 3 vorgestellte DGGM-Rahmenwerk an dieser Grundstruktur orientieren.

\subsection{Von Daten zu ,Big Data“}

Grundsätzlich wird unter einem DGGM ein Geschäftsmodell verstanden, in dem Daten eine Kernressource in der Wertarchitektur des Geschäftsmodells darstellen (Hartmann et al. 2016). Zur Beschreibung zentraler Dateneigenschaften im digitalen Zeitalter wird in der Geschäftsmodellliteratur oftmals auf die sog. „4 Vs“ von Big Data verwiesen (volume, variety, velocity, veracity) (siehe z. B. Schroeder 2016; Wiener et al. 2020). In diesem Zusammenhang ist allerdings darauf hinzuweisen, dass die Verfügbarkeit von bzw. der Zugang zu großen Datenmengen (volume) für nicht wenige Organisationen schon seit der Einführung von relationalen Datenbanken in den 1970er-Jahren ,nichts mehr wirklich Neues“ darstellt (Pigni et al. 2016). Zudem ist ,bigness“ alleine ein wenig geeignetes Kriterium zur aussagekräftigen Abgrenzung von Big Data, was auch die heutzutage fast schon inflationäre Nutzung dieses Begriffes erklären vermag (Schroeder 2016). Außerdem sind die Unsicherheiten (veracity) und die damit verbundenen Herausforderungen, die große Datenvolumina hervorrufen können (z. B. die erschwerte Erkennung von Verzerrungen und/oder systematischen Fehlern in den Daten), zwar nicht grundsätzlich vernachlässigbar, aber eher technischer und nicht sozio-technischer Natur. Gerade aus Perspektive der Wirtschaftsinformatik führt daher die Volumenfrage an sich nicht zu entscheidenden Veränderungen. (Im Folgenden vernachlässigen wir daher das Datenvolumen und sprechen ,nur“ von Daten und nicht von Big Data).

Auch wenn das insgesamt verfügbare Datenvolumen neue Ausmaße erreicht (Hartmann et al. 2016), sind die damit einhergehenden, eher qualitativen Veränderungen von größerer Bedeutung (Pigni et al. 2016). Diese werden unter den Schlagwörtern ,variety“ und ,velocity“ diskutiert und können als wesentliche Treiber des Datenvolumens gesehen werden (Pigni et al. 2016). Die zunehmende Datenvielfalt (variety) ist darauf zurückzuführen, dass neben internen Daten (z. B. aus ERPSystemen) auch unternehmensexterne Daten (z. B. aus sozialen Medien) in großem Umfang zur Verfügung stehen; dass neben strukturierten Daten auch häufig unstrukturierte Daten (z. B. Twitter-Tweets) relevant sind; und dass letztere nicht nur textbasiert sind, sondern oftmals auch Multimedia-Daten (z. B. Videos eingebettet in Tweets) enthalten können. Die bedeutsamste Neuerung in Bezug auf Big Data ist aber vermutlich die zunehmende Verfügbarkeit und Verarbeitung von Echtzeit-Datenströmen (velocity) (Pigni et al. 2016). Entsprechende Datenströme werden sowohl von smarten Maschinen oder smarten Dingen (z.B. RFID-Daten, IoT-Sensordaten) als auch von Menschen selbst (z. B. Posts oder Tweets in sozialen Medien) generiert oder entstehen durch Mensch-Maschine-Interaktionen (z. B. Log-Daten, Mobilitäts- 
daten, Tracking-Daten). Vor diesem Hintergrund erscheint die Bezeichnung von Menschen als ,walking data generator[s]“ (McAfee und Brynjolfsson 2012, S. 63) als durchaus angebracht.

Darüber hinaus betonen Pigni et al. (2016), dass Big Data und die damit verbundenen Dateneigenschaften (,4 Vs“) nicht per se von Nutzen für Organisationen sind. Diesbezüglich verweisen Pigni et al. (2016) zum Beispiel auf Unternehmen, die zwar reich an Daten, aber arm an Informationen sind. Folglich stellt sich die Frage, welche Merkmale von Daten bzw. Datenbeständen diese potenziell ,wertvoll““ machen. Günther et al. (2017) führen den Wert von Daten(beständen) insbesondere auf zwei sozio-technische Dateneigenschaften zurück: zum einen Portabilität - also die Nutzbarkeit von bestehenden Daten in verschiedenen Anwendungskontexten; und zum anderen Interkonnektivität - also die Verknüpfbarkeit von Daten mit anderen Datenbeständen, so dass durch die Verknüpfung und/oder Kombination neue Erkenntnisse entstehen. Ob und wie mit Daten Wert geschaffen und realisiert werden kann, ist schlussendlich jedoch eine Frage der konkreten Ausgestaltung des jeweiligen, unternehmensspezifischen DGGM.

\section{Datengetriebene Geschäftsmodelle (DGGM)}

Wie in Abschnitt 2.2 bereits angedeutet, verstehen wir ein DGGM als ein Geschäftsmodell, in dem Daten eine Kernressource darstellen und somit eine bedeutende Rolle in der Wertarchitektur des Geschäftsmodells einnehmen (Hartmann et al. 2016). Ob die jeweilige Organisation diese Daten selbst generiert, einkauft oder über einen Partner erhält, ist dabei zunächst irrelevant. Auch ist es für die Abgrenzung zwischen DGGM und traditionellen Geschäftsmodellen zunächst unbedeutend, ob Daten als interne Ressource betrachtet werden oder ob sie als externer Faktor für einen zu erbringenden Service dienen - also den Objektfaktor darstellen, an dem die Dienstleistung erbracht wird.

Neben den in Abschnitt 2 eingeführten konzeptuellen Grundlagen zu Geschäftsmodellen und wesentlichen Dateneigenschaften nennt und diskutiert die einschlägige Literatur mittlerweile eine Vielzahl von Konzepten zur Beschreibung, Analyse und/oder Gestaltung von DGGM. In diesem Zusammenhang werden wir im Folgenden zunächst drei unterschiedliche Typen von DGGM vorstellen. Im Anschluss daran werden wir auf ausgewählte Konzepte eingehen, die wir für eine weitergehende Charakterisierung von DGGM im Allgemeinen und die drei DGGM-Typen im Speziellen als nützlich erachten, und diese in ein konzeptuelles Rahmenwerk integrieren.

\subsection{Typen von DGGM}

In der Forschungsliteratur erfolgt eine Unterscheidung zwischen drei Typen von Big Data-Geschäftsmodellen (Schroeder 2016; siehe auch Wiener et al. 2020): Datennutzer (user), Datenlieferanten (supplier) und Datenbefähiger (facilitator). Auch wenn sich diese Typologie explizit auf „Big Data“ bezieht, so lässt sie sich unseres Erachtens eins-zu-eins (d.h. ohne weitere Anpassungen) auf DGGM im Allgemei- 
Tab. 1 DGGM-Typen. (In Anlehnung an Schroeder 2016 und Wiener et al. 2020)

\begin{tabular}{|c|c|}
\hline Typ & Typspezifische Wertschöpfungstätigkeiten (exemplarisch) \\
\hline$\overline{\text { Datennutzer }}$ & $\begin{array}{l}\text { Nutzung von Daten zur: } \\
\text { Automatisierung und/oder Unterstützung von Entscheidungen } \\
\text { Verbesserung von internen Aktivitäten und Prozessabläufen } \\
\text { Erweiterung von bestehenden Produkten und Services } \\
\text { Entwicklung von neuartigen Produkten/Services }\end{array}$ \\
\hline Datenlieferanten & $\begin{array}{l}\text { Sammlung, Aufbereitung und Verkauf von (Primär-)Daten } \\
\text { Entwicklung und Vermarktung von Datenprodukten (ggf. inklusive der Bereitstellung } \\
\text { von Hardware zur kundenseitigen Datensammlung) }\end{array}$ \\
\hline Datenbefähiger & $\begin{array}{l}\text { Angebot von datenbezogenen Analyse- und Beratungsleistungen (z. B. über die } \\
\text { „Cloud“) } \\
\text { Vertrieb von Dateninfrastrukturlösungen (Hard- und Software) }\end{array}$ \\
\hline
\end{tabular}

nen anwenden. Wie der Name schon sagt, geht es bei Datennutzern in erster Linie um die eigentliche Nutzung von verfügbaren Datenbeständen zu Zwecken der Wertschöpfung, d.h. zur Schaffung und Realisierung von Werten. Hierbei kann der Fokus sowohl auf der internen Wertschöpfung (z.B. Nutzung von Daten bzw. Datenanalyseergebnissen zur kontinuierlichen Verbesserung von internen Prozessabläufen) und/oder auf der externen Wertschöpfung (z. B. datengetriebene Entwicklung von neuartigen Kundenprodukten bzw. die Weiterentwicklung entsprechender Produkte) liegen. Hingegen liegt der Wertschöpfungsfokus von Datenlieferanten bzw. Datenbefähigern insbesondere auf der Unterstützung von Datennutzern durch die Versorgung mit relevanten Daten oder Datenprodukten (Lieferanten) bzw. durch das Angebot von unterstützenden Datenservices oder Dateninfrastrukturlösungen (Befähiger). Tab. 1 gibt einen Überblick über die drei DGGM-Typen und beschreibt diese exemplarisch anhand von typspezifischen Wertschöpfungstätigkeiten.

An dieser Stelle ist anzumerken, dass Datennutzer die Hauptkunden von Datenlieferanten und Datenbefähigern sind. Datenbefähiger können aber auch Datenlieferanten oder andere Datenbefähiger unterstützen (Schroeder 2016, S. 9).

Darüber hinaus ist anzumerken, dass die drei hier eingeführten DGGM-Typen in der Praxis nur selten in ihrer idealtypischen Reinform auftreten. Zum Beispiel werden Datenlieferanten oftmals auch datennahe Services anbieten, um die nachgefragten Daten in der vom Kunden (Datennutzer) benötigten Form bereitstellen zu können. Nichtsdestotrotz erscheint es wahrscheinlich, dass in einem konkreten DGGM immer einer der drei Typen dominiert und somit eine Einordnung in die hier vorgestellte Typologie möglich ist.

\subsection{Konzeptuelles Rahmenwerk für DGGM}

Entlang der vier Geschäftsmodelldimensionen konzeptualisieren wir zentrale Merkmale, die sich für eine weitergehende Charakterisierung von DGGM eignen. Diese Merkmale sind grundsätzlich auf alle drei DGGM-Typen anwendbar, wenngleich es auch typspezifische Schwerpunkte geben kann. Das im Folgenden dargestellte Rahmenwerk eignet sich daher, konkrete DGGM spezifischer zu beschreiben und dient damit der Ergänzung allgemeiner Geschäftsmodell-Rahmenwerke. 
Wertversprechen Hartmann et al. (2016) unterscheiden hinsichtlich des Wertangebots eines DGGMs zwischen Daten, Informationen/Wissen und traditionellen Produkten/Services. Auf dieser Basis differenzieren wir zwischen vier wesentlichen DGGM-Wertangeboten: (1) Daten als Produkt, (2) datengetriebene Services, (3) datenbezogene Infrastrukturlösungen und (4) hybride Leistungsbündel bestehend aus einem physischen Produkt im weiteren Sinne (wie z.B. einer Werkzeugmaschine) und darauf aufbauenden, datengetriebenen Services. Während datengetriebene Services letztendlich für alle drei DGGM-Typen (siehe Abschnitt 3.1) relevant sind, lassen sich die anderen drei Wertangebote schwerpunktmäßig jeweils einem DGGMTyp zuordnen. Rohdaten und andere Datenprodukte (inklusive Lösungen zur kundenseitigen Datensammlung) werden von Datenlieferanten angeboten; Dateninfrastrukturlösungen (z. B. zur Speicherung und/oder Analyse von großen Datenmengen) werden von Datenbefähigern bereitgestellt; und hybride Leistungsbündel werden typischerweise von Datennutzern vermarktet.

Wertarchitektur Wie bereits eingangs betont, stellen Daten per Definition eine Kernressource in der Wertarchitektur eines DGGM dar. Zur weitergehenden Beschreibung dieser Kernressource sind unter anderem die vielfältigen internen und externen Datenquellen zu betrachten, die hier zum Tragen kommen können (variety). Hinsichtlich interner Datenquellen kann nach Hartmann et al. (2016) zwischen bereits existierenden Daten (die z. B. in unternehmensinternen ERP-Systemen oder anderen Anwendungssystemen vorgehalten werden) und selbstgenerierten Daten (die z.B. über Tracking-Systeme oder Crowdsourcing-Initiativen generiert werden) unterschieden werden. Demgegenüber können sich externe Datenquellen auf akquirierte bzw. zugekaufte, kundenseitig bereitgestellte oder frei verfügbare Datenbestände beziehen. Letztere können weiter unterteilt werden in öffentliche Daten (bereitgestellt z.B. durch Einrichtungen der öffentlichen Verwaltung), über standardisierte APIs zugängliche Daten aus sozialen Medien (z. B. Twitter) und sogenannte ,webcrawled“ Daten, die mithilfe von programmierten Auswertungsroutinen aus dem Internet gewonnen werden können. Im Falle von (Echtzeit-)Datenströmen (velocity) kann zudem zwischen maschinen- und menschgenerierten Daten sowie zwischen Datenströmen, die sich aus Mensch-Maschine-Interaktionen ergeben, unterschieden werden (siehe Abschnitt 2.2).

Weitere relevante, bereits in Abschnitt 2.2 eingeführte Dateneigenschaften sind die Portabilität und Interkonnektivität. Im Gegensatz zu dedizierten Daten, die meist auch wegen ihrer Nichtverfügbarkeit für andere Unternehmen in Datennutzer-Geschäftsmodellen einen entscheidenden Wettbewerbsvorteil bieten können, sind die Portabilität und Interkonnektivität ausgesprochen wichtige Eigenschaften in einem Datenlieferanten-Geschäftsmodell, weil gerade diese Eigenschaften die Schaffung zielgerichteter Datenprodukte für eine große Zahl an Kunden und Kundengruppen ermöglichen. In einem solchen DGGM kommt es darauf an, dass aus vormals möglicherweise dedizierten Ressourcen so genannte „Multi-usage“Ressourcen (Osterwalder et al. 2020, S. 255) werden.

Neben der Kernressource Daten umfasst die Wertarchitektur eines DGGM auch entsprechende Kernaktivitäten. Um diese zu konzeptualisieren, greifen wir zum einen auf eine Taxonomie von Hartmann et al. (2016) und zum anderen auf von 
Pigni et al. (2016) identifizierte Archetypen wertschaffender, datenbezogener Aktivitäten zurück:

1. Datengenerierung: Erzeugung oder Zugänglichmachung von Datenbeständen und -strömen (auch in Echtzeit) aus verschiedenen Quellen (Pigni et al. 2016; Hartmann et al. 2016);

2. Datenaggregation: zielgerichtete Auswahl bzw. Zusammenführung/Kombination von Datenbeständen und -strömen (Pigni et al. 2016; Hartmann et al. 2016);

3. Datenbeschaffung: systematische Beschaffung von externen Datenbeständen, im Sinne eines Daten-Sourcing (Hartmann et al. 2016);

4. Datenanalyse: Analyse von Datenbeständen zur Entscheidungsunterstützung in deskriptiver, prädiktiver oder präskriptiver Form einschließlich der zugehörigen Aufbereitungen (z. B. Datenvisualisierung) (Pigni et al. 2016; Hartmann et al. 2016); sowie

5. Entwicklung datengetriebener Services: Weiterentwicklung von Daten und Analyseergebnisse zu Diensten, die in der Regel über eine reine Daten- oder Ergebnisbereitstellung hinausgehen (Pigni et al. 2016).

Aufbauend hierauf lässt sich die Wertarchitektur der drei DGGM-Typen weiter charakterisieren: Bei Datennutzern stehen die Kernaktivitäten Datenanalyse (4) und Serviceentwicklung (5) im Vordergrund. Je nachdem, ob sie über die Daten selbst verfügen, ausschließlichen Zugang zu diesen haben oder sie bei Datenlieferanten zukaufen, sind auch die ersten drei Aktivitäten von Bedeutung. Datenlieferanten hingegen haben einen Fokus auf die Datengenerierung und -aggregation $(1,2)$, beschaffen aber möglicherweise auch ergänzende Daten (3). Datenbefähiger schließlich können bei allen fünf Aktivitäten unterstützen, in der Regel durch Dienstleistungen, aber auch durch Produkte/Infrastruktur, die diese Aktivitäten erheblich erleichtern. Die für die DGGM-Typen jeweils typischen Aktivitäten weisen bereits auf Abhängigkeiten zu Schlüsselpartnern im Wertnetzwerk eines DGGM hin.

Wertnetzwerk Zwischen den DGGM-Typen können verschiedene Abhängigkeiten bestehen (Schroeder 2016, S. 9). Aus der Perspektive ihrer jeweiligen Wertnetzwerke, d.h. ihrer Schlüsselpartner, lassen sich diese wie folgt charakterisieren. Während Datennutzer auf Datenlieferanten und/oder Datenbefähiger als typische Schlüsselpartner zurückgreifen, können Datenlieferanten und -befähiger grundsätzlich auch weitgehend eigenständig (d. h. ohne Schlüsselpartnerschaften mit DGGMbasierten Unternehmen) agieren. Denkbar ist bei Datenlieferanten, dass sie selbst einen Teil der Daten von anderen Datenlieferanten beziehen und/oder auch Unterstützung von Datenbefähigern in Anspruch nehmen. Ebenso können Datenbefähiger zur Erbringung ihrer Leistungen auf andere Datenbefähiger als Schlüsselpartner setzen.

Wertrealisierung Im Hinblick auf die Wertrealisierung verweisen Wixom und Ross (2017) auf drei grundsätzliche Arten der Datenmonetarisierung: Unter ,improving " verstehen sie das Verbessern von internen Prozessen und Entscheidungen unter Verwendung von Daten, was sich sowohl positiv auf die Kostenstruktur ei- 
nes Geschäftsmodells auswirken kann (z.B. durch die Steigerung der Prozesseffizienz) als auch der Erschließung neuer Umsatzquellen dienen kann. Mit dem sog. „wrapping“ bezeichnen Wixom und Ross das Umhüllen von bereits bestehenden, physischen Produkten und klassischen Services mit neuen, datengetriebenen Services (wie z. B. das Angebot von Predictive Maintenance-Services auf der Basis von Maschinendaten), wohingegen unter ,selling“ das eigentliche Verkaufen von Daten(-produkten) oder darauf aufbauenden Datenservices zu verstehen ist.

Während die ersten beiden Datenmonetarisierungsarten (Verbessern und Umhüllen) insbesondere für Datennutzer relevant sind, bezieht sich die dritte Monetarisie-

Tab. 2 Konzeptuelles DGGM-Rahmenwerk

\begin{tabular}{|c|c|c|}
\hline $\begin{array}{l}\text { Dimension: } \\
\text { Element(e) }\end{array}$ & Ausprägungen & Referenz(en) \\
\hline $\begin{array}{l}\text { Wertversprechen: } \\
\text { Produkte/Services }\end{array}$ & $\begin{array}{l}\text { Typen von Wertangeboten: } \\
\text { Daten als Produkt } \\
\text { Dateninfrastrukturlösungen } \\
\text { Datengetriebene Services } \\
\text { Hybride Leistungsbündel (Kombination aus physischem } \\
\text { Produkt und datengetriebenen Services) }\end{array}$ & $\begin{array}{l}\text { In Anlehnung an } \\
\text { Hartmann et al. } \\
(2016)\end{array}$ \\
\hline $\begin{array}{l}\text { Wertarchitektur: } \\
\text { Kernressourcen }\end{array}$ & $\begin{array}{l}\text { Datenquellen (variety): } \\
\text { Interne Daten (bereits existierende vs. selbstgenerierte Da- } \\
\text { ten) } \\
\text { Externe Daten (akquirierte vs. vom Kunden bereitgestellte } \\
\text { vs. frei verfügbare Daten) }\end{array}$ & $\begin{array}{l}\text { Hartmann et al. } \\
(2016)\end{array}$ \\
\hline & $\begin{array}{l}\text { Datenströme (velocity): } \\
\text { Maschinengeneriert } \\
\text { Menschengeneriert } \\
\text { Generiert durch Mensch-Maschine-Interaktionen } \\
\text { Weitere Dateneigenschaften: } \\
\text { Portabilität } \\
\text { Interkonnektivität }\end{array}$ & $\begin{array}{l}\text { Günther et al. } \\
\text { (2017) }\end{array}$ \\
\hline $\begin{array}{l}\text { Wertarchitektur: } \\
\text { Kernaktivitäten }\end{array}$ & $\begin{array}{l}\text { Datenbezogene, wertschaffende Aktivitäten: } \\
\text { Datengenerierung } \\
\text { Datenaggregation } \\
\text { Datenbeschaffung } \\
\text { Datenanalyse (deskriptiv, prädiktiv und präskriptiv; inklusive } \\
\text { Datenaufbereitung/-visualisierung) } \\
\text { Entwicklung datengetriebener Services }\end{array}$ & $\begin{array}{l}\text { In Anlehnung an } \\
\text { Hartmann et al. } \\
\text { (2016) und Pigni } \\
\text { et al. (2016) }\end{array}$ \\
\hline $\begin{array}{l}\text { Wertnetzwerk: } \\
\text { Schlüsselpartner }\end{array}$ & $\begin{array}{l}\text { Schlüsselpartner mit DGGM (siehe Abschnitt 3.1): } \\
\text { Datenlieferanten } \\
\text { Datenbefähiger } \\
\text { Weitere Schlüsselpartner ohne DGGM }\end{array}$ & $\begin{array}{l}\text { Schroeder (2016) } \\
\text { und Wiener et al. } \\
(2020)\end{array}$ \\
\hline $\begin{array}{l}\text { Wertrealisierung: } \\
\text { Kostenstruktur \& } \\
\text { Umsatzquellen }\end{array}$ & $\begin{array}{l}\text { Datenmonetarisierung (ökonomischer Wertbeitrag): } \\
\text { Verbessern } \\
\text { Umhüllen } \\
\text { Verkaufen }\end{array}$ & $\begin{array}{l}\text { Wixom und Ross } \\
\text { (2017) }\end{array}$ \\
\hline
\end{tabular}


rungsart (Verkaufen) sowohl auf Datenlieferanten als auch auf Datenbefähiger. Hier kann eine Unterscheidung danach herbeigeführt werden, ob das Wertversprechen eines DGGM in erster Linie auf dem Angebot von Daten(-produkten) als immaterielle Ware fußt (Lieferant) oder allen voran auf das Angebot von datenbezogenen Services, wo Daten den Objektfaktor darstellen, fokussiert (Befähiger). Insbesondere in Bezug auf das Verkaufen ist hinzuzufügen, dass entsprechende DGGM oftmals mit Wertangeboten verbunden sind, bei denen die Kunden mit ihren (persönlichen) Daten für diese Angebote ,zahlen“ (z. B. Rüdian und Vladova 2021). „Pay-withdata“-Ansätze sind in der Regel mit zwei- oder mehrseitigen Plattform-Geschäftsmodellen verknüpft. Zur Vertiefung verweisen wir auf Schüritz et al. (2017), die unterschiedliche Typen solcher Ansätze bei mehrseitiger Erlösgenerierung aufzeigen und diskutieren.

Tab. 2 gibt einen zusammenfassenden Überblick entlang der Dimensionen und Elemente des konzeptuellen DGGM-Rahmenwerks.

\section{Praxisbeispiele für datengetriebene Geschäftsmodelle}

Zur Illustration der drei DGGM-Typen (siehe Abschnitt 3.1) sowie zur Veranschaulichung, wie das in Abschnitt 3.2 eingeführte Rahmenwerk zur weitergehenden Charakterisierung von DGGM genutzt werden kann, stellen wir im Folgenden exemplarisch für jeden Typ je ein Unternehmen vor: KAESER, Shell Telematics und Celonis.

KAESER ist ein Beispiel für einen Datennutzer. Das Unternehmen mit Hauptsitz in Coburg ist einer der weltweit führenden Anbieter von Druckluftsystemen und mit insgesamt rund 7000 Mitarbeitern in über 140 Ländern aktiv (Kaeser 2021b). Beim zentralen Wertversprechen des DGGM von KAESER handelt es sich um hybride Leistungsbündel bestehend aus physischen Maschinen (insbesondere Druckluftkompressoren und komplementäre Maschinen, z. B. zur Speicherung und Verteilung von Druckluft) und darauf aufbauenden, datengetriebenen Services (z. B. zur Predictive Maintenance). Um entsprechende Services anbieten zu können, greift KAESER auf unterschiedliche Datenquellen zurück. In diesem Zusammenhang sind allen voran Maschinenzustandsdaten (z. B. Luftdruck, Temperatur und Vibrationen) zu nennen, die über in die Kompressoren integrierte Sensoren erfasst (maschinengeneriert) und über eine Industrial Internet of Things (IIoT)-Lösung in Fast-Echtzeit an eine zentrale SAP HANA-Datenbank am Firmensitz übermittelt werden (Bock et al. 2019). In Abhängigkeit des kundenspezifischen Betreibermodells handelt es sich hierbei um externe Daten (traditionelles Modell, bei dem der Kunde das Druckluftsystem kauft und weitgehend eigenständig betreibt) oder um interne Daten (Modell, in dem KAESER den operativen Systembetrieb vollständig übernimmt und dem Kunden ,Druckluft zum Festpreis“ anbietet). Daten zum aktuellen Maschinenzustand werden zudem mit historischen Daten zur Maschinennutzung und zum Energieverbrauch sowie mit SAP-Stammdaten (z. B. zu Kunden, Ersatzteilen und Servicetechnikern) kombiniert. Darüber hinaus greift das Unternehmen zur Analyse der individuellen Rahmenbedingungen von Neukunden auf zum größten Teil menschengenerierte Log-Daten zurück. Diese umfassen neben Daten zum kundenspezifischen Druckluftbedarf auch 
Daten zur Luftqualität innerhalb und außerhalb (d.h., in der unmittelbaren Umgebung) der Fertigungsstätte, wo das Druckluftsystem zum Einsatz kommen soll (Bock et al. 2019).

Als Kernaktivitäten in der Wertarchitektur des DGGM von KAESER sind insbesondere die Generierung bzw. Zugänglichmachung von Maschinenzustandsdaten (durch Sensortechnik und zugehörige IIoT-Lösungen), die Aggregation von Daten (z. B. durch die Kombination von aktuellen Maschinendaten mit historischen Daten) und die kontinuierliche Analyse entsprechender Daten einschließlich einer adäquaten Datenvisualisierung (z. B. in Form von Management-Dashboards) zu nennen. Bezüglich der Datenanalyse kommen sowohl deskriptive Analyseformen (z. B. zum zentralen Monitoring der kundenseitig installierten und über die ganze Welt verteilten Druckluftsysteme) als auch prädiktive Analyseformen (z.B. zum rechtzeitigen Anstoßen von notwendigen Maschinenwartungsprozessen) zum Einsatz (Bock et al. 2019). Außerdem arbeitet KAESER ständig an der Entwicklung von (neuen) datengetriebenen Services zur Unterstützung von Kunden beim kosteneffizienten Betrieb von Druckluftsystemen bis hin zur vollständigen Übernahme des operativen Systembetriebs durch KAESER im Rahmen des sog. „SIGMA AIR UTILITY“Betreibermodells (Kaeser 2021a).

Ermöglicht wird das DGGM von KAESER durch strategische Partnerschaften mit zwei Datenbefähigern: zum einen mit dem ERP-Softwarehersteller SAP, von dem unter anderem die zentrale In-Memory Datenbank und die IIoT-Lösung bereitgestellt wird; und zum anderen mit der Deutschen Telekom, die maßgeblich an der Entwicklung und dem Betrieb einer mobilen IT-Lösung zur Unterstützung von KAESER-Mitarbeitern bei der Analyse des kundenseitigen Druckluftbedarfs beteiligt ist (Bock et al. 2019).

Hinsichtlich der Wertrealisierung fokussiert das KAESER-DGGM insbesondere auf das sog. Umhüllen des Kernprodukts (Druckluftkompressoren) mit datengetriebenen Services. Darüber hinaus gewinnen Daten zunehmend an Bedeutung im Hinblick auf das Verbessern von internen und externen Prozessabläufen (z. B. automatisierte Ersatzteilbestellung und Koordination von Servicetechnikern) sowie als zentraler Input bei der internen Weiterentwicklung von bestehenden Produktlösungen und/oder der Entwicklung von Produktinnovationen (Bock et al. 2019).

Unter der Bezeichnung Shell Telematics firmiert ein von Shell entwickeltes DGGM, das dem Typ Datenlieferant entspricht. Durch Shell Telematics bereitgestellte On-Board-Dongles (OBD), die mit dem Fahrzeug über die OBD-Buchse verbunden sind, werden Fahrzeugdaten in Echtzeit aufgezeichnet und Kunden in einem Cloud-basierten Onlineportal zur Verfügung gestellt. Daten werden also als Produkt angeboten. Es handelt sich dabei um externe Daten, die über Mensch-Maschine-Interaktionen (bzw. Fahrer-Fahrzeug-Interaktionen) generiert und mit externen Geo-Daten kombiniert werden. Die generierten Daten werden zudem verknüpft mit Transaktionsdaten der Shell-Tankkarte (,Shell Card“), die als interne Daten betrachtet werden können. Wie für einen Datenlieferanten typisch, handelt es sich um Daten hoher Portabilität und Interkonnektivität, die folglich von Shell TelematicsKunden vielfältig genutzt werden können. Shell Telematics bietet hier neben den in einem Onlineportal bereitgestellten Daten noch weitergehende datengetriebene Services zum Flottenmanagement an (Shell 2021a, b). 
Da Shell Telematics den Fahrzeugdatenstrom über den Dongle zugänglich macht, handelt es sich um Datengenerierung. Durch Verknüpfung mit vorhandenen Kundentransaktionsdaten und Geo-Daten findet auch Datenaggregation statt. Beides ist typisch für einen Datenlieferanten. Über das Leistungsspektrum eines reinen Datenlieferanten gehen die Datenanalysen und Dienstentwicklungen hinaus. Dabei ist neben deskriptiven Analysen, einschließlich ihrer Visualisierung, auch von prädiktiven (z. B. Schätzungen von Zeitangaben) und präskriptiven (z. B. kraftstoffeffiziente Streckenwahl) auszugehen. Als Schlüsselpartner im Wertnetzwerk von Shell Telematics ist Geotab zu nennen, ein Anbieter einer offenen Flottenmanagement-Plattform, der gegenüber Shell Telematics als Datenbefähiger einzustufen ist (Shell 2021a, b).

Am Beispiel von Shell Telematics zeigt sich, dass die Grenzen zwischen einem Datenlieferanten und einem Datennutzer fließend sein können. Typisch für Datenlieferanten erfolgt die Wertrealisierung durch das Verkaufen eines Datenprodukts, das im Fall von Shell Telematics in verschiedenen „Paket-Versionen“ erhältlich ist. Die Nutzung des Datenprodukts wird über eine monatliche Abonnementgebühr (zuzüglich Dongle-Miete und Installationskosten) abgerechnet. Das DGGM von Shell Telematics zeigt aber auch Ansätze des Umhüllens, da der bisherige Tankkarten-Dienst datengetrieben erweitert wird. Insgesamt ist das durch Shell Telematics ermöglichte Wertversprechen jedoch in erster Line auf das neue Datenprodukt zurückzuführen, so dass wir den Datenlieferanten-Typ im Vordergrund sehen.

Celonis ist eines der wenigen deutschen „Unicorn“-Startups (d.h. ein junges Unternehmen mit einer Marktbewertung von über einer Milliarde US-Dollar), das in 2019 einen Marktwert von 2,5 Mrd. US-Dollar erreichte (Höpner et al. 2019; Treyz 2020) und ein Paradebeispiel für einen Datenbefähiger darstellt. Das Unternehmen entwickelt und vertreibt Software, die es Kundenunternehmen ermöglicht, große Datenbestände zum Zweck des Process Mining zu analysieren, ohne dabei selbst über tiefe Process Mining-Expertise verfügen zu müssen. Neben Datennutzern als Kunden bietet Celonis seine Leistungen auch anderen Datenbefähigern (,Celonis for Consulting“") zur Nutzung bei der Erbringung eigenständiger, datenbezogener Dienstleistungen an (Celonis 2021).

Die angebotene Dateninfrastrukturlösung in Form der Softwarelösung „Celonis Process Mining“ (aktuelle Produktbezeichnung: Celonis Execution Management System [EMS]) unterstützt die automatisierte Identifikation und Modellierung von Prozessen (,discovery“) sowie die Analyse von Prozessen zur Konformitätsprüfung von Ist- gegenüber Soll-Prozessmodellen (,,conformance“), zur Prozessverbesserung (,enhancement“") bis hin zur Echtzeitprozessunterstützung durch Integration in operative Systeme (Peters und Nauroth 2019).

Typisch für einen Datenbefähiger ist, dass es nicht darum geht, eigene Daten oder Daten für eigene Zielsetzungen zu analysieren, sondern diese als Objektfaktor, an denen eine Leistung erbracht wird, zu betrachten. Es handelt sich folglich um externe Daten. Aus der Perspektive der unterstützten Kundenunternehmen sind es in der Regel interne Daten, die durch Mensch-Maschine-Interaktionen bei der Nutzung von transaktionalen Anwendungssystemen im Unternehmen entstehen und in der Form von Log-Daten digitale Spuren hinterlassen.

Diese Log-Daten können aus unterschiedlichsten Systemen stammen und werden durch Process Mining verknüpft und zu einem Prozessmodell verdichtet, so 


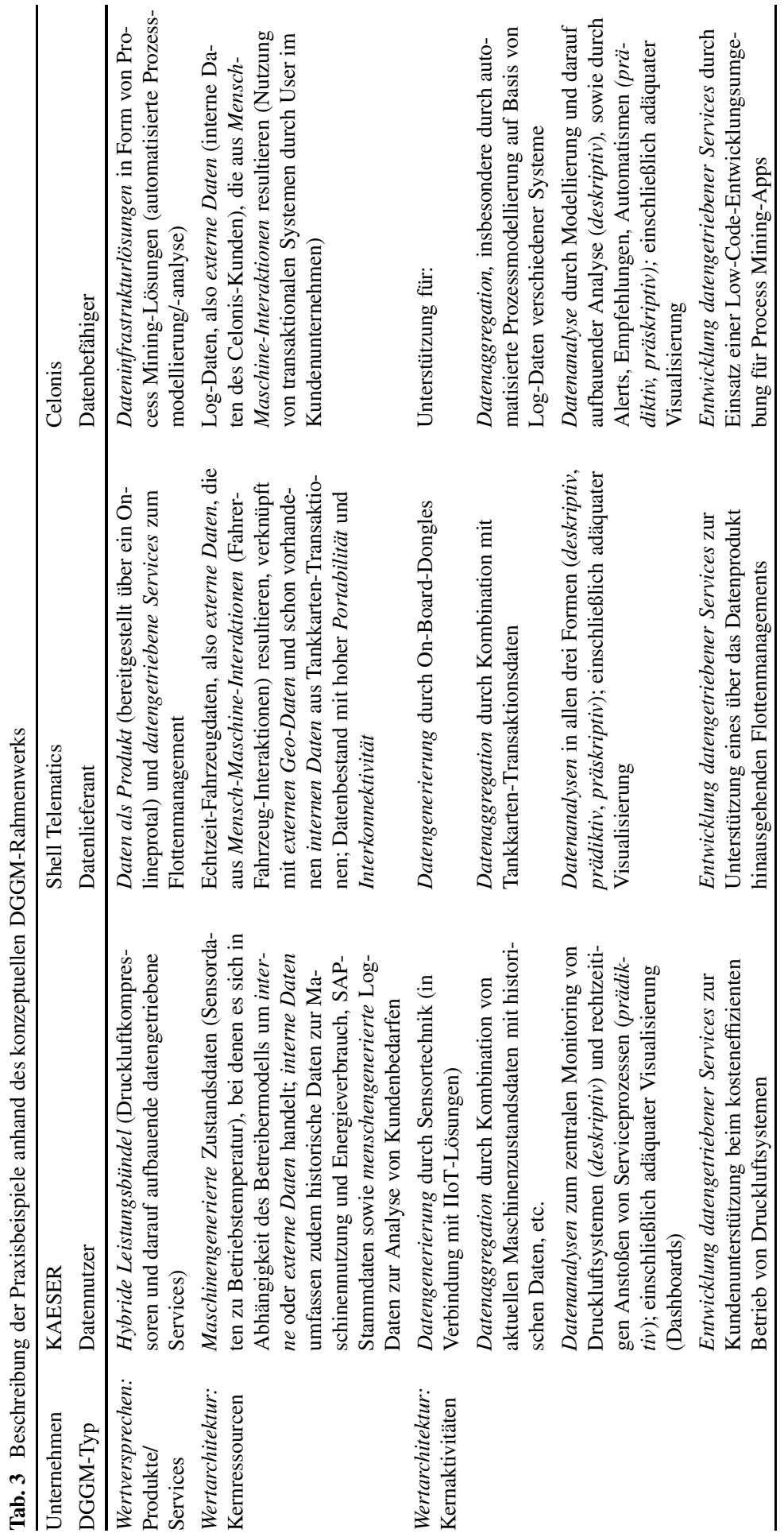




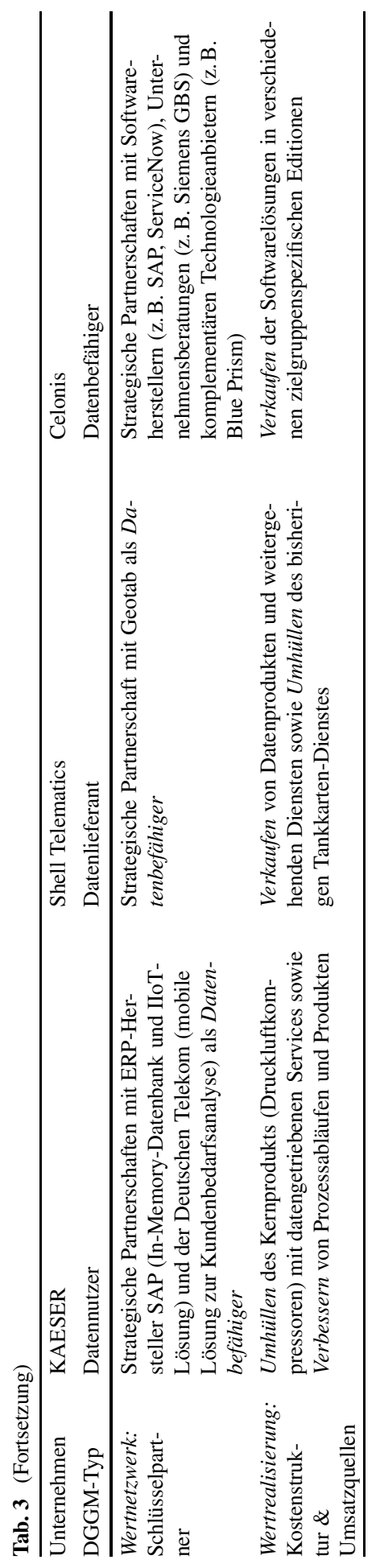


dass Datenaggregation stattfindet. Darauf aufbauende Unterstützung gilt primär der Datenanalyse, die in erster Linie deskriptiv ist, aber auch in operative Systeme integriert werden kann und dort Trigger-basiert, weitergehende Automatisierung ermöglicht, z. B. Warnungen auslöst und Handlungsempfehlungen unter Nutzung von Geschäftsregeln und maschinellem Lernen gibt. Folglich werden auch prädiktive und präskriptive Analysen unterstützt. Die Visualisierung ist ebenfalls Teil der Softwarelösung. Mittels der Low-Code-Entwicklungsumgebung „Celonis Studio“ können Kunden zudem in der Entwicklung datengetriebener Services unterstützt werden (Celonis 2021).

Als wesentliche Schlüsselpartner von Celonis sind ausgewählte Standardsoftwarehersteller (z. B. SAP, ServiceNow) zu nennen, aus deren Systemen bei Celonis-Kundenunternehmen die zu analysierenden Log-Daten stammen und in die hinein eine Trigger-basierte Integration der Process Mining-Analyseergebnisse vollzogen wird. Aber auch Beratungs- und Systemhäuser (z. B. Siemens Global Business Services) sowie andere Technologieanbieter, die über komplementäre Technologien verfügen (z. B. der Robotic Process Automation-Anbieter Blue Prism), gehören in das Celonis-Wertnetzwerk. Celonis bietet seine Systeme in unterschiedlichen zielgruppenspezifischen Editionen am Markt an und folgt damit dem klassischen Ansatz des Verkaufens, um die geschaffenen Werte zu realisieren (Celonis 2021).

Tab. 3 fasst die unternehmensspezifischen Merkmale der DGGM von KAESER, Shell Telematics und Celonis zusammen und stellt sie entlang der fünf Dimensionen des oben eingeführten DGGM-Rahmenwerks gegenüber.

\section{Diskussion und Forschungsausblick}

Die im vorliegenden Grundlagenbeitrag vorgestellten DGGM-Typen sowie das im Beitrag abgeleitete DGGM-Rahmenwerk können Praktikern bei der Einordnung, Analyse und Beschreibung von bereits bestehenden bzw. noch zu entwickelnden DGGM unterstützen und Forschern zugleich als konzeptuelle Grundlage für neue Studien dienen. Vor diesem Hintergrund möchten wir im Folgenden, basierend auf der einschlägigen Forschungsliteratur, auf eine Auswahl von unseres Erachtens vielversprechenden, vorwiegend thematischen Ansatzpunkten für zukünftige Forschungsarbeiten zu DGGM eingehen.

Hinsichtlich der DGGM-Typen weisen erste Literaturanalysen darauf hin, dass sich existierende Studien bislang größtenteils auf die Untersuchung von Datennutzern fokussiert haben (Wiener et al. 2020). Insofern wäre ein verstärkter Forschungsfokus auf die Untersuchung von Datenlieferanten und -befähigern im Allgemeinen wünschenswert. Darüber hinaus wäre es hilfreich, wenn zukünftige Studien auch verstärkt das Zusammenspiel zwischen den drei DGGM-Typen betrachten würden. Hier ergeben sich unter anderem interessante Fragestellungen bezüglich der Ausgestaltung der Schnittstellen zwischen unterschiedlichen DGGM-Typen. Zum Beispiel stellt sich für einen Datennutzer die Frage, inwieweit er datenbezogene Wertschöpfungsaktivitäten selbst (,,in-house“) erbringen sollte und für welche Aktivitäten er unter welchen Rahmenbedingungen auf Datenlieferanten (z. B. zur Generierung und Aggregation von Daten) und/oder auf Datenbefähiger (z.B. zur Datenanalyse und 
-visualisierung) zurückgreifen sollte. Ein interessanter Kontext zur Untersuchung des Zusammenspiels von verschiedenen DGGM-Typen könnte z. B. die Erforschung von Datenplattformökosystemen darstellen (vgl. Kramberg und Heinzl 2021).

Bei genauerer Betrachtung der Geschäftsmodellliteratur fällt zudem auf, dass sich Studien oftmals auf die Erforschung der DGGM von Start-ups beschränken (z.B. Hartmann et al. 2016; Schüritz et al. 2017). Ob bzw. inwieweit sich die Ergebnisse dieser Studien auf etablierte Unternehmen übertragen lassen, ist zumindest fraglich, da Start-ups bei der Gestaltung ihrer DGGM typischerweise einen „Greenfield“-Ansatz verfolgen können (d.h. sie müssen keine Rücksicht auf bestehende Strukturen und Geschäftsbeziehungen nehmen) (Hartmann et al. 2016). Hinzu kommt, dass sich verfügbare Forschungsarbeiten typischerweise auf die statische Analyse von DGGM begrenzen (Wiener et al. 2020). Im Gegensatz hierzu sind Studien, die die Entwicklung eines DGGM oder die Transformation hin zu einem solchen Geschäftsmodell aus einer (dynamischen) Prozessperspektive untersuchen, noch weitgehend „Mangelware“. Eine nennenswerte Ausnahme ist die Studie von Dremel et al. (2017), die die datengetriebene Transformation des traditionellen Geschäftsmodells der AUDI AG beschreibt und auf dieser Basis ein dreistufiges Prozessmodell (,advancing, enabling, and leveraging") vorschlägt. Folglich wäre aus Forschungssicht ein verstärkter Fokus auf die Entwicklung oder Transformation von DGGM aus einer Prozessperspektive erstrebenswert (vgl. Hoßbach et al. 2016; Vial 2019). Diesbezüglich könnte das im vorliegenden Beitrag eingeführte, konzeptuelle DGGM-Rahmenwerk zum Beispiel zur Beschreibung von verschiedenen Entwicklungs- bzw. Transformationsstufen herangezogen werden. In diesem Zusammenhang ist zugleich die noch weitgehend fehlende Methodenunterstützung für ein systematisches Management von DGGM-Entwicklungs- und Transformationsprozessen auffällig (vgl. Förster et al. 2021).

Im Hinblick auf die Gestaltung und den Betrieb von DGGM verweist die Forschungsliteratur auf eine Vielzahl von rechtlichen und ethischen Fragestellungen, die eine tiefergehende Auseinandersetzung und Untersuchung erfordern. Zum Beispiel weisen Studien darauf hin, dass derzeit in Bezug auf den Datenschutz erhebliche Unterschiede zwischen Europa und den USA bestehen (Schroeder 2016; Wiener et al. 2020), was die Umsetzung von DGGM durch europäische bzw. deutsche Unternehmen zum Teil nachhaltig erschwert und letztendlich zu strategischen Wettbewerbsnachteilen für diese Unternehmen führen könnte (Loebbecke and Picot 2015). Ein konkretes Problem in diesem Zusammenhang ist zum Beispiel der Umgang mit Daten mit hoher sozio-technischer Portabilität (Günther et al. 2017). Ein möglicher Lösungsansatz zum datenschutzkonformen Umgang mit solchen Daten könnte die Einrichtung von öffentlichen und privatwirtschaftlichen Datentreuhandstellen darstellen (Arlinghaus et al. 2021). Ein weiterer Ansatzpunkt für Datennutzer könnte die umfangreichere Auslagerung von datenbezogenen Wertschöpfungsaktivitäten an Datenlieferanten im Allgemeinen sein. Genauer gesagt können rechtliche und auch ethische Probleme im Umgang mit sensiblen Daten - insbesondere mit solchen mit hoher sozio-technischer Portabilität - für Datennutzer zumindest teilweise gelöst werden, wenn sich diese eines Datenlieferanten bedienen und dabei auf die explizite Lieferung von Daten zugunsten der Lieferung von Analyseergebnissen (z.B. in Form des Erreichens eines Schwellwertes) verzichten. So können beispiels- 
weise Gesundheitsboni bei Krankenversicherungen an das Erreichen von Fitnesszielen geknüpft werden, ohne dass Tracking-Daten von Geräten der Versicherten (z. B. Smartphones, Fitness-Tracker) direkt oder indirekt über Gesundheitsplattformen (z. B. Apple Health, Fitbit) zum Versicherungsunternehmen gelangen müssten. Wird alles außerhalb der Sphäre der Versicherung (Datennutzer) verarbeitet und nur sehr datensparsam die Zielerreichung mit Zustimmung des Versicherten ,gemeldet“, entledigt sich der Datennutzer möglicherweise vieler rechtlicher und auch ethischer Probleme. Dass dieses Modell aber nur funktioniert, weil große Datenlieferanten in oftmals intransparenten Verflechtungen Daten sammeln und aggregieren, ohne dass Konsumenten noch verstehen können, welchen Datenverwendungen sie zustimmen, ist die moralische bzw. ethische Kehrseite dieser „Umgehungspraxis“ via externer Datenlieferanten (Wulf und Betz 2021).

Des Weiteren sollte noch erwähnt werden, dass sich existierende Studien zu DGGM bislang nahezu ausschließlich auf den ökonomischen Wertbeitrag - also auf die Monetarisierung von Daten durch Effizienzsteigerungen und/oder die Erschließung neuer Umsatzquellen - fokussieren (z. B. Schüritz et al. 2017; Wixom und Ross 2017). Auch wenn dieser Fokus vor dem Hintergrund der derzeit weit verbreiteten Betrachtungsweise von Daten als ,das neue Öl“ nicht verwunderlich ist, so wäre es dennoch wünschenswert, dass zukünftige Studien auch zunehmend den potenziellen sozialen Wertbeitrag von (Big-)Daten und entsprechenden Geschäftsmodellen betrachten (Günther et al. 2017). Idealerweise finden entsprechende Studien auch Lösungsansätze, wie sich die beiden - also ökonomischer und sozialer Wertbeitrag im Rahmen von DGGM vereinen lassen. Ein Beispiel ist das norwegische Telekommunikationsunternehmen Telenor, das große Mengen an (kommerziellen) Mobilfunkdaten bereitstellt und diese in Kooperation mit öffentlichen Einrichtungen und weiteren nichtstaatlichen Organisationen nutzt, um die Verbreitung von Pandemien, neuen Krankheiten und Naturkatastrophen vorherzusagen (Telenor 2021).

Abschließend kann festgehalten werden, dass die Forschung zu DGGM in den letzten Jahren sehr erfreuliche Fortschritte gemacht hat; aber auch, dass es nach wie vor eine Vielzahl an offenen Forschungsfragen und -problemen gibt, die es noch zu adressieren gilt. Wir hoffen, dass wir mit diesem Grundlagenartikel einen Beitrag zur Systematisierung und Weiterentwicklung des Forschungsfelds leisten konnten und dass andere Forscherinnen und Forscher hieraus neue Ideen und Impulse für zukünftige Forschungsarbeiten schöpfen können.

Funding Open Access funding enabled and organized by Projekt DEAL.

Open Access Dieser Artikel wird unter der Creative Commons Namensnennung 4.0 International Lizenz veröffentlicht, welche die Nutzung, Vervielfältigung, Bearbeitung, Verbreitung und Wiedergabe in jeglichem Medium und Format erlaubt, sofern Sie den/die ursprünglichen Autor(en) und die Quelle ordnungsgemäß nennen, einen Link zur Creative Commons Lizenz beifügen und angeben, ob Änderungen vorgenommen wurden.

Die in diesem Artikel enthaltenen Bilder und sonstiges Drittmaterial unterliegen ebenfalls der genannten Creative Commons Lizenz, sofern sich aus der Abbildungslegende nichts anderes ergibt. Sofern das betreffende Material nicht unter der genannten Creative Commons Lizenz steht und die betreffende Handlung nicht nach gesetzlichen Vorschriften erlaubt ist, ist für die oben aufgeführten Weiterverwendungen des Materials die Einwilligung des jeweiligen Rechteinhabers einzuholen. 
Weitere Details zur Lizenz entnehmen Sie bitte der Lizenzinformation auf http://creativecommons.org/ licenses/by/4.0/deed.de.

\section{Literatur}

Al-Debei MM, Avison D (2010) Developing a unified framework of the business model concept. Eur J Inf Syst 19(3):359-376

Arlinghaus T, Kus K, Kajüter P, Teuteberg F (2021) Datentreuhandstellen gestalten: Status quo und Perspektiven für Geschäftsmodelle. HMD 58(3). https://doi.org/10.1365/s40702-021-00727-x

Bock M, Wiener M (2017) Towards a taxonomy of digital business models-conceptual dimensions and empirical illustrations. ICIS 2017 proceedings. https://aisel.aisnet.org/icis2017/Strategy/ Presentations/19/. Zugegriffen: 26. Febr. 2021

Bock M, Wiener M, Gronau R, Martin A (2019) Industry 4.0 enabling smart air: digital transformation at Kaeser compressors. In: Urbach N, Röglinger M (Hrsg) Digitalization cases. Springer, Cham, S 101-117

Brynjolfsson E, Hitt LM, Kim HH (2011) Strength in numbers: how does data-driven decisionmaking affect firm performance? Social Science Research Network, Rochester

Celonis (2021) Homepage Celonis. https://www.celonis.com/. Zugegriffen: 26. Febr. 2021

Chen H, Chiang RHL, Storey VC (2012) Business intelligence and analytics: from big data to big impact. MISQ 36(4):1165-1188. https://doi.org/10.2307/41703503

Dremel C, Wulf J, Herterich MM et al (2017) How AUDI AG established big data analytics in its digital transformation. MIS Q Executive 16(2):81-100

Forbes (Hrsg) (2020) The 2020 world's most valuable brands. https://www.forbes.com/the-worlds-mostvaluable-brands/. Zugegriffen: 26. Febr. 2021

Förster M, Bansemir B, Roth A (2021) Innovation von datengetriebenen Geschäftsmodellen - Oft probiert, häufig gescheitert? Ein Vorgehensmodell zur Wertrealisierung von Daten. HMD 58(3). https://doi. org/10.1365/s40702-021-00730-2

Gassmann O, Frankenberger K, Csik M (2014) The business model navigator: 55 models that will revolutionise your business. Pearson Education Limited, Harlow, New York

Günther WA, Rezazade Mehrizi MH, Huysman M, Feldberg F (2017) Debating big data: a literature review on realizing value from big data. J Strateg Inf Syst 26(3):191-209

Hartmann PM, Zaki M, Feldmann N, Neely A (2016) Capturing value from big data-a taxonomy of datadriven business models used by start-up firms. Int J Oper Prod Manag 36(10):1382-1406

Höpner A, Kapalschinski C, Müller A (2019) Process-Mining-Spezialist: Deutsches Start-up Celonis erreicht Bewertung von 2,5 Mrd. Dollar. https://www.handelsblatt.com/technik/it-internet/processmining-spezialist-deutsches-start-up-celonis-erreicht-bewertung-von-2-5-milliarden-dollar/ 25253078.html. Zugegriffen: 22. Febr. 2021

Hoßbach N, Wiener M, Saunders C (2016) The unfolding of value sources during online business model transformation. J Bus Models 4(2):22-41. https://doi.org/10.5278/ojs.jbm.v4i2.1623

Kaeser (2021a) Betreibermodell: Sigma Air Utility. https:/www.kaeser.de/produkte/betreibermodellsigma-air-utility/. Zugegriffen: 26. Febr. 2021

Kaeser (2021b) Unternehmen - Kaeser Kompressoren. https://www.kaeser.de/unternehmen/. Zugegriffen: 26. Febr. 2021

Kramberg P, Heinzl A (2021) Datenplattformökosysteme. HMD 58(3). https://doi.org/10.1365/s40702021-00716-0

Loebbecke C, Picot A (2015) Reflections on societal and business model transformation arising from digitization and big data analytics: A research agenda. The Journal of Strategic Information Systems 24(3):149-157. https://doi.org/10.1016/j.jsis.2015.08.002

McAfee A, Brynjolfsson E (2012) Big data: the management revolution. Harv Bus Rev 90(10):60-68

Österle H, Blessing D (2005) Ansätze des Business Engineering. HMD 42(1):7-17

Osterwalder A, Pigneur Y (2010) Business model generation: a handbook for visionaries, game changers, and challengers. Flash Reproductions, Toronto

Osterwalder A, Pigneur Y, Tucci CL (2005) Clarifying business models: origins, present, and future of the concept. Comm Assoc Inform Syst 16(1):1-25. https://doi.org/10.17705/1CAIS.01601

Osterwalder A, Pigneur Y, Etiemble F (2020) The invincible company. Wiley, Hoboken

Peters R, Nauroth M (2019) Process-Mining: Geschäftsprozesse: smart, schnell und einfach. Springer Fachmedien Wiesbaden, Wiesbaden 
Pigni F, Piccoli G, Watson R (2016) Digital data streams: creating value from the real-time flow of big data. Calif Manage Rev 58(3):5-25

Rotella P (2012) Is data the new oil? https://www.forbes.com/sites/perryrotella/2012/04/02/is-data-thenew-oil/. Zugegriffen: 26. Febr. 2021

Rüdian S, Vladova G (2021) Kostenfreie Onlinekurse nachhaltig mit personalisiertem Marketing finanzieren - Ein Vorschlag zur synergetischen Kombination zweier datengetriebener Geschäftsmodelle. HMD 58(3). https://doi.org/10.1365/s40702-021-00720-4

Schroeder R (2016) Big data business models: challenges and opportunities. Cogent Soc Sci 2(1):1-15

Schüritz R, Seebacher S, Dorner R (2017) Capturing value from data: revenue models for data-driven services. In: HICSS 2017 proceedings https://doi.org/10.24251/HICSS.2017.648

Shell (2021a) Shell Telematics. https://www.shell.de/geschaefts-und-privatkunden/shell-card/digitaleservices-und-sicherheit/shell-telematics.html. Zugegriffen: 18. Jan. 2021

Shell (2021b) Shell telematics for fleet management. https:/www.shell.co.uk/business-customers/shellfuel-card/shell-telematics.html. Zugegriffen: 26. Febr. 2021

Telenor (2021) Big data for social good. https://www.telenor.com/innovation/research/big-data-for-socialgood/. Zugegriffen: 26. Febr. 2021

Treyz C (2020) German unicorn Celonis on why startups should keep universities close. https://sifted.eu/ articles/celonis-alex-rinke/. Zugegriffen: 22. Febr. 2021

Veit D, Clemons E, Benlian A et al (2014) Business models. Bus Inf Syst Eng 6(1):45-53. https://doi.org/ 10.1007/s12599-013-0308-y

Vial G (2019) Understanding digital transformation: a review and a research agenda. J Strateg Inf Syst 28(2):118-144

Wiener M, Saunders C, Marabelli M (2020) Big-data business models: A critical literature review and multiperspective research framework. J Inf Technol 35(1):66-91

Wixom BH, Ross JW (2017) How to monetize your data. MIT Sloan Manage Rev 58(3):10-13

Wulf N, Betz S (2021) Daten-Ökosysteme wider Willen: Herausforderungen des Pay-as-you-live-Geschäftsmodells im Kontext deutscher Krankenversicherungen. HMD 58(3). https://doi.org/10.1365/ s40702-021-00719-X 